\title{
Why did Engineering Students Choose to Study in China? 工科学生缘何来华留学?
}

\author{
Guoyang Zhang 张国洋 \\ Shanghai Jiao Tong University, China 上海交通大学，中国 \\ Jiabin Zhu 朱佳斌 \\ Shanghai Jiao Tong University, China 上海交通大学, 中国
}

\begin{abstract}
China is currently the third-largest destination country for international students in the world. Among the international students in China, the number of those in engineering schools is increasing most rapidly. Based on the "Push-Pull" theory (Altbach, 1998) and a three-stage decision-making theory (Ma, 2017), this research explored the factors influencing international students' choices of engineering schools in China. Twenty-two international students in engineering schools at a leading Chinese university were interviewed. Qualitative analyses of the interview data suggested that the participants were attracted to China by the availability of scholarships, ranking of the host university, and opportunities for personal growth and professional development. Meanwhile, it was found that positive relationships between the students' home countries and China, inadequate educational and research resources in home countries, and higher tuition fees and educational costs involved in studying in Western countries also influenced their decision-making process. Based on the findings, suggestions and recommendations were proposed for further enhancement of learning experiences of international students in engineering disciplines in China.
\end{abstract}

摘要: 中国已经成为全球第三大留学目的国, 其工科留学生数量增速最快。本研 究以囊括宏观因素与微观因素的 “推拉” 理论和国际学生择校三阶段理论为指导, 采用质性研究方法, 以 $\mathrm{S}$ 校为研究平台, 一对一地访谈了来自亚、欧、美、非洲 的 22 名工科学生, 旨在探索形成工科学生来华留学 “热潮” 的原因。研究发现, 可获得奖学金、雄厚的工科实力、促进个人发展是影响他们来华留学的主要因素, 友好的两国关系、母国教育与研究资源的匮乏、西方国家昂贵的留学费用在择校 过程中也起到了一定作用。针对中国高校吸引力不足的问题, 研究提出了增加英 文授课课程, 提高教师队伍专业水平; 加大海外招生宣传力度, 优化招生手段; 发挥奖学金杜杆作用, 吸引高层次工科留学生等建议。 
Keywords: engineering students, studying in China, decision-making process, qualitative research 关键词: 工科学生, 留学中国, 择校过程, 质性研究

全球国际学生的数量从 1999 年的 200 万人增加至 2018 年的 500 多万人, 增量庞 大且增速迅猛（OECD, 2018）。近些年学生的跨国流动开始出现 “反循环” 现 象, 即美国、英国等传统留学生输入大国所占的高等教育国际市场份额开始下降, 而中国、新加坡等传统的留学生输出国开始加入高等教育国际市场的竞争行列

（De Wit \& Altbach, 2018; Ruby, 2009）。自 2010年以来，中国实施《留学中国 计划》、完善中国政府奖学金资助体系、打造 “留学中国” 品牌、启动世界一流 大学和一流学科建设、发起 “一带一路” 倡议等措施更是加快了中国高等教育国 际化的步伐。国际教育协会（Institute of International Education）发布的国际学生 流动性研究报告显示: 中国大陆自 2015 年至今一直是全球第三大留学目的国, 仅次于美国、英国, 约占有 $10 \%$ 的市场份额。其中, 工科来华留学生在 2002 年 仅有 2,442 人, 随后以 $20 \%$ 的年均增速递增。 2017 年, 工科来华留学生共计 58,090 人, 其学历生占比、获奖学金留学生占比均高居第一（教育部国际合作与 交流司，2003-2018）。近二十年来，为何形成了工科学生来华留学的 “热潮” ? 为了回答这一引人深思的问题, 本研究在文献梳理的基础上, 运用质性研究方法 深入探索了影响工科学生来华留学的因素, 呈现了工科学生来华留学的决策过程, 以期剖析中国及中国高校在吸引工科留学生上的优势与不足, 为中国更好地发展 工科来华留学生教育提供一定的参考与借鉴。

\section{文献综述}

\section{国际学生流动的推拉理论}

国际学生流动的推拉理论最早由美国社会学家 Lee(1966) 提出, 用以分析移 民的迁移规律。Altbach（1998）利用该理论分析了学生的跨国流动, “推拉”即母 国的“推动因素”和东道国的“拉力因素”。此后，该理论框架被广泛地应用于国际 学生流动动因的研究之中, “推动因素”包括母国教育质量差、入学机会有限、社 会动乱、经济发展落后等, “拉力因素”包括东道国教育质量好、可获得的奖学金、 就业机会多、国际化程度高等（Chen \& Barnett, 2000; Mazzarol \& Soutar, 2002）。 然而一些学者发现推拉理论从宏观层面上解释了国际学生的流动原因, 却忽略了 微观层面上学生个体的主观能动性, 他们的调查发现: 学生出于追求学术职业、 增加经济资本、方便未来移民、学习他国文化等原因也会选择出国留学 (Chao et al., 2017; Chen, 2007; Nghia, 2019）。

\section{国际学生择校的三阶段理论}

除了运用推拉理论分析国际学生择校的原因之外, 还有学者探索了国际学生 择校的具体决策过程与阶段。Mazzarol 和 Soutar (2002) 提出国际学生在择校时 会经历选择是否出国、决定留学国家、篮选高校三个阶段, 即留学择校三阶段理 论。随后, Chen (2007) 的研究发现, 学生可能在择校时直接选择特定高校, 
他重新定义了择校三阶段: 选择是否出国、笁选高校或学位项目、确定最终选择。 Ma（2017）则调查了在两个差异性较大城市就读的留学生的留学动机, 添加了 篮选城市或者机构作为第二阶段。

\section{来华留学生留学动机与择校过程的相关研究}

近些年来, 来华留学生数量激增, 而关于留学生留学动机和决策过程的研究 却少之又少。一些学者发现影响留学生来华留学的重要因素有中国的经济发展水 平高、合适的学位项目、教育质量好、两国学历互认、母国教育落后等（Ding, 2016; Lu \& Tian, 2018; Wen \& Hu, 2018; 李立国, 陈岗, \& 赫文婧, 2015; 宋华盛 \& 刘莉, 2014; 孙慧莉 \& 李忠明, 2017）。Ma（2017）运用扎根理论的方法, 在访谈 42 名来华留学生的基础上, 提出了一个将推拉理论与留学三阶段理论结合起来 分析留学生具体择校阶段与过程的框架。她发现国际学生择校的过程十分复杂, 大致可以分为三个阶段：第一阶段-一选择中国阶段, 影响因素有中国光明的发展 前景、学习汉语、可获得的奖学金、体验新生活等; 第二阶段-一选择城市或机构 阶段, 影响因素有城市的历史底蕴、高校的教学质量等; 第三阶段--决定阶段, 学生在衡量以上所有因素的基础上做出最终选择。

总的来说, 现有研究多关注整个来华留学生群体, 缺少对工科留学生的研究, 更缺乏对其留学动机及其择校过程的清晰描述。随着中国工程教育实力不断提升、 工科来华留学生日益增多, 深入剖析影响工科学生来华留学的因素具有重要的现 实意义。本研究借鉴 Ma (2017) 的研究发现, 在囊括微观因素与宏观因素的推 拉理论和国际学生择校三阶段理论的指导下对工科来华留学生的择校过程进行分 析。一方面, 已有的择校三阶段理论为分析来华留学生的择校过程提供了一个初 步框架, 另一方面, 在此基础上, 在每个阶段中分析与该阶段的选择相关的因素 既体现了与推拉理论相关的宏观因素, 又体现了个体动机与影响因素。借助这个 理论框架, 本文深入剖析工科来华留学生的择校过程, 试图为中国在来华留学生 教育领域进一步发挥现有优势、改善招生与培养过程, 从而吸引优秀的工科来华 留学生源提供实证依据。

\section{研究方法}

本研究以工科实力较强、工科来华留学生较多的 $\mathrm{S}$ 校为研究平台, 采用质性研究 的方法, 借鉴 Ma（2017）的研究发现, 在囊括微观因素与宏观因素的推拉理论 和国际学生择校三阶段理论的指导下，设计了 “您为什么选择来华留学？”、

“您为什么不去欧美国家留学?”、“您为什么选择来上海留学？”、“您为什 么选择来 $\mathrm{S}$ 校就读? ”、“哪个因素是最重要的影响因素呢? ”等访谈问题, 运 用一对一半结构化访谈的方式访谈了来自巴基斯坦、尼泊尔、法国、美国、伊朗、 伯利兹、塞内加尔、孟加拉国、荷兰的 22 名即将毕业的硕士与博士研究生, 他 们就读的专业有机械工程、电气工程、土木工程、材料科学与工程等。小规模质 性研究存在参与者有限的问题, 因此在招募留学生的过程中, 本研究综合考虑学 生的性别、生源地、学位层次 (博士、硕士) 等不同因素进行分层抽样, 以确保 样本具有一定的代表性。

研究者将英文版访谈录音上传至 “讯飞听见”语音转录网站 (https://www.iflyrec.com) 进行转录, 也对转录文本进行了多轮校对以确保文本 
的准确性。最后, 研究者使用 ATLAS.ti 质性数据分析软件, 对质性资料进行了 三个阶段的编码：开放式编码、主轴式编码、选择性编码（Strauss \& Corbin, 1990）。整个数据分析的过程是循环往复的, 最后对所有的编码和类属进行梳理 和整合，提取了三个不同主题（阶段）下面的具体类属。

\section{研究发现}

\section{第一阶段：选择中国}

\section{可获得的奖学金}

本研究中的所有被访者都获得了政府奖学金（中国或上海市）或 $\mathrm{S}$ 校留学生 奖学金, 他们都表示获得全额公费奖学金是来华留学的主要原因。奖学金不仅肯 定了他们的学业表现, 而且使他们获得了经济支持, 能够更加专注于学业和研究。 Porter 是一名来自巴基斯坦的硕士生，他说:

“我拿到了中国的全额奖学金, 就不用像德国的朋友一样兼职了, 我可以一 心一意地做研究, 实现我的学习和研究目标。”

此外，欧美国家提供的奖学金难以获得、金额较少且等待奖学金结果的时间 较长, 而中国的公费奖学金较容易获得且申请流程简单, 这间接促使工科学生选 择来华留学。例如 Baker 说到:

“本科的时候，美国只能给我提供免 75\% 学费的奖学金, 这是远远不够的。 后来我就汶览网页寻找机会, 发现中国有一个第一年学习汉语, 之后可以学习工 程的学位项目, 我就报名了, 幸运地拿到了全额奖学金, 硕士又拿到了上海市政 府奖学金，就更愿意留在中国读书了。”（Baker，伯利兹）

\section{相对较低的受教育成本}

大部分工科留学生表示英国、德国、美国等欧美国家昂贵的学费、生活费让 他们望而却步, 而中国相对较低的受教育成本却能让他们接受性价比很高的教育。 例如, 一些被访者说到:

“我申请了很多国家, 但我发现英国等国家的留学费用太高了。尽管德国不 需要付学费, 但生活费用我也负担不起。而中国既提供奖学金, 生活成本又低。”

(Nanda，尼泊尔）

“在荷兰读研究生花费多还要缴税, 在中国读书既有奖学金又不用缴税。整 体而言, 中国生活费用也比较低。”（Daniel, 荷兰)

“美国的学费很贵，如果我投资了 5 万美元，我家可以负担得起，但是我却 不能得到同等的回报，这对我和我家人是不公平的。”（Paddy，巴基斯坦）

\section{稳定友好的两国关系}

很多被访者认为两国平等友好的关系不仅意味着他们在中国不会受到歧视， 中国人会友好地对待他们, 而且意味着两国会有多方面的政治、经济合作，有助 于个人的未来发展。对中国情有独钟的 Paddy 说: 
“中国和巴基斯坦的关系一直很好，我想去一个受欢迎的国家……因为一带 一路倡议，中国在巴基斯坦投资的资金是最多的……后无论回国就业还是在中 国就业，都是很好的选择。”

塞内加尔是西非第一个与中国签署一带一路文件的国家, 来自该国的 Simon 提到:

“因为一带一路，中国在我们国家投资建设了很多的基础设施，两国友好的 关系让我觉得来中国学习对我未来发展很有前景。”

\section{促进个人职业发展}

超过一半的工科学生表示他们希望通过来华留学拓展自身的知识和技能以胜 任未来的工作。例如, Payne 讲述了知识储备不足的经历, 他说:

“我在巴基斯坦读硕士，我也是巴基斯坦另一所高校的老师，学生们会问我 一些数据分析、数据处理、做实验的问题, 而我却不知道怎么回答他们, 我的老 师也不知道怎么回答他们。我想只有去国外更好的高校学习更多新的知识和技能, 才能够做好教书育人的工作。”（Payne，巴基斯坦）

Baker 认为: “本科毕业时, 我们学习的知识都很泛化。我想精通本领域, 希 冀毕业时可以具备充足的知识和能力以找到好的工作。”（Baker，伯利兹）

\section{中国工业的蓬勃发展}

接近一半的工科留学生认为中国工业正在蓬勃发展、实力较强, 有着先进的 技术与丰富的就业机会。Nash 提到中国人在尼泊尔拥有很多房地产和建筑公司, 认为 “中国有先进的工此技术和实力” , 希望 “学习这些先进的技术, 应用到本 国的发展之中”; 巴基斯坦的 Parker 也说到:

“巴基斯坦的工业水平比较低, 就业机会也很少。而中国近些年来工业一直 在蓬勃发展, 有很多大的工厂, 有很多的就业机会, 我想未来在中国找一份工程 师的工作。”

\section{较为简单的申请程序}

一些被访者认为申请中国高校的流程相对简单、申请材料较少、等待时间较 短。例如, 博士生 Inman 讲述了申请母国博士可能要走的 “艰辛历程” :

“我们国家的博士招生制度和中国相似，需要先通过三科笔试，然后面试。 四到六个教授坐在我的对面，用很久的时间询问我的研究和实验。作为硕士生， 我怎么可能会清晰地回答这些问题？”

他放弃了在国内读博, 选择中国的部分原因是由于留学生申请流程较为简单:

“申请中国就不一样，我只要提交英语成绩证明、成绩单、体检证明、推荐 信，再进行不到十五分钟的面试就可以了。”（Inman,伊朗）

来自巴基斯坦的硕士生 Peter 和博士生 Parish 都提到相比于欧美国家, 申请 来华留学 “流程较容易”、“花费时间较少”、“等待录取结果时间较短” 等。 


\section{学习汉语与中国文化}

本研究中的工科留学生就读的学位项目均为英文授课项目, 申请标准只规定 了学生托福或雅思的基准分, 未限制其汉语水平。少部分留学生或因个人兴趣、 或受家庭朋友的影响、或为便利个人生活, 他们期望学习汉语与中国文化, 体验 一种不同的生活方式。Fidel 是一位来自法国的第三代华裔，“从未来过中国”、 “对中国人和中国文化的好奇”、“周围中国同学和家人的影响”是他来中国留 学的一些原因; Adel在决定来中国留学之前, 提前体验了两周的中国生活, 他说:

“我和妻子都很喜欢中国, 想了解中国文化……因为可能需要购物、在饿了 么定外卖、上淘宝买东西, 我认为学习汉语中的一些基本生活用语是十分必要 的。” (Adel, 美国)

\section{来自他人的影响}

少数被访者表示, 亲人曾经去过欧美国家和中国, 对不同国家的生活有一定 的了解, 他们选择国家的时候会考虑来自亲人的意见和建议。最为典型的例子是 Pamela, 她说:

“我当时申请了很多的学校, 还拿到了欧盟的伊斯拉莫奖学金, 我当时挺想 去欧洲的。但是, 我来自传统的穆斯林家庭, 要绝对服从父亲。我父亲觉得我叔 叔在欧洲过的生活很不好, 他自己也来过中国几次, 觉得中国很安全, 因此让我 来中国读书。” 回顾四年的博士生涯, 她认为来中国留学是她人生中做过的最正 确的决定之一。

\section{第二阶段：选择 $\mathbf{S}$ 校}

\section{突出的全球大学排名表现}

绝大部分工科来华留学生表示, S 校在 QS、US NEWS 等全球大学排行榜上 位居前列, 甚至超过了欧美很多著名工程院校, 部分工科专业也进入了全球五十 强, 这都表示 $\mathrm{S}$ 校拥有雄厚的工程实力。如下面受访者所说, 排名是影响工科学 生选择 $\mathrm{S}$ 校的重要因素。

“ $S$ 校的排名很好, 特别是电院的排名也在全球前 50 , 这是我选择 $S$ 校的一 个决定性因素。”（Petra，巴基斯坦）

“我自己在网上随意汶览的时候发现 $S$ 校不仅在中国有很好的排名, 在世界 上也是。我主修的学科, $S$ 校的全球大学排名也很好。” (Page, 巴基斯坦)

“在法国的时候, 我们有一些双学位项目, 中国学生来到我们学校学习。他 们说 $S$ 校是很好的大学, 排名也很好。我也觉得这是一个很好的机会。”（Fidel, 法国） 


\section{雄厚的学术研究实力}

$\mathrm{S}$ 校拥有大量的科研经费、先进的可供学生使用的研究设备, 以及具备卓越 研究能力的教师, 其工科学术研究实力在中国首屈一指, 更位居世界前列, 吸引 了诸多工科留学生。Neil 说:

“我在搜寻大学的时候发现, $S$ 校给学生的研究机会竟如此之多, 竟然还让 研究生使用近百万美元的研究设备。我想做更多的研究, 能够使用这些实验设备 是我来 $S$ 校的主要原因。”（Neil，尼泊尔）

一些学生认为尽管母国的科研水平在不断上升, 但还存在学术氛围寡淡的问 题, 相比于中国高校, 依旧是望尘莫及, 这反推着他们来华留学。Neil 还说:

“中国和欧美国家的研究生都在做研究, 而我们国家的研究生和本科生没有 太大的区别, 很难接触到学术研究。老师们也忙于拉工程项目, 而不是沉浸于学 术研究。”

\section{良好的大学声誉}

不少工科留学生都提到, $\mathrm{S}$ 校的学科声誉、教学声誉、研究声誉都很好。这 不仅意味着他们在 $S$ 校可以接受到良好的教育, 而且意味着他们能获得有价值的 学位。例如, Paul 本科就读于华中科技大学, 毕业后工作了三年, 为了提升知识 技能, 他申请了比本科学校排名更好的 $\mathrm{S}$ 校和复旦大学, 因 “ $S$ 校工科很受欢 迎”、“ $\mathrm{S}$ 校的工科学位更有价值” 选择了 $\mathrm{S}$ 校（Paul, 巴基斯坦）; Philip 通过 朋友们的介绍了解到“ $S$ 校有极好的声誉, 学习环境也很好。” (Philip, 巴基斯 坦)

\section{合适的学位项目}

很多被访者认为, 他们倾向于选择与他们本科专业相契合的学位项目或符合 个人兴趣的学位项目。由于他们无汉语基础, 在寻找学位项目时只能选择全英文 授课项目, S 校则是中国极少向外国研究生提供英文授课的学校之一。Nanda 讲 到:

“我本科学的是桥梁工程, 也想继续学习这个专业。我在大学排行榜上看到 了清华大学, 但是他们没有适合的英文授课项目, 而我看到 $S$ 校有合适的学位项 目, 我就来了。”（Nanda, 尼泊尔）

值得一提的是, S 校和法国、美国的一些院校创办了一些双学位项目, 参加 这些学位项目的学生可以拿到双学位。Fidel 所在的法国里昂国立应用科学学院 刚好与 $\mathrm{S}$ 校有合作，他来中国的目的之一是 “拿到两所顶级工科院校的学位”。

\section{来自他人的影响}

一些学生在申请 $\mathrm{S}$ 校的时候在一定程度上会受到朋友或导师的影响。他们的 朋友会讲述自己在 $\mathrm{S}$ 校的就读经历并向受访者推荐 $\mathrm{S}$ 校, 受访者相信来自朋友的 经验比官方宣传更为真实。Petra 在访谈中提到:

“我加入了一个由在华留学的巴基斯坦人组成的近三四千人的群, 学长学姐 们向我们推荐了 $S$ 校。” 


\section{Peter 说:}

“我的一些朋友已经在西安交通大学和 $S$ 校读书了, 他们告诉我 $S$ 校很好, 建议我申请 $S$ 校。当时正好有一个一带一路的学位项目, 我就申请了 $S$ 校。”

(Peter, 巴基斯坦)

据少数工科学生描述, 申请 $\mathrm{S}$ 校需要先联系导师, 如果他们对导师的研究方 向感兴趣且双方交流无障碍，他们就会选择 $\mathrm{S}$ 校。Inman 讲述了自己来 $\mathrm{S}$ 校的经 历:

“我在网上搜索教授时发现, 我导师的研究方向和我的研究方向很契合, 然 后就通过邮件进行了愉快的交流。他在新西兰、澳大利亚、中国都有教职, 但他 大部分时间呆在中国，他让我申请 $S$ 校。”（Inman，伊朗）

然而, 少数被访者提及在华留学会遇到一些挑战。比如, 虽然 $\mathrm{S}$ 校采用了全 英文授课，但是全英文授课的课程数量有限，且教师英文授课水平欠佳。这些挑 战可能会影响他们向母国学生推荐来华留学, 也可能会使他们转向他国继续深造。 例如, Paul 说:

“我认为每个人都应该修读至少一门编程课程, 为他们的研究工作做准备。 然而许多编程课程都是中文的, 我们并不能听懂。我必须自己修读 edX、 Coursera 等网站上的课程才能做研究。”

Nanda 提到:

“我们会和老师产生沟通隔阂，也许他们可以用中文更好地传达工程知识， 但是用英语演讲时, 我们不能很好地理解他们的观点……结合我个人兴趣, 我末 来想去美国继续读博士。”

\section{恰到好处的学制}

部分工科学生认为 $\mathrm{S}$ 校的研究生学制很合理, 给予了他们较为充足的时间做 研究。欧美的硕士研究生只有一年的学制, 并没有足够的时间学习专业知识、做 研究; 清华大学的博士研究生需要学习一年的汉语。相比而言, 他们认为 $S$ 校两 年半的硕士生学制和四年的博士生学制恰到好处。例如 Neil 说:

“我也申请了英国的学校，但通过和教授的聊天中发现英国的学制只有一年， 大部分时间在上课写论文, 几乎没时间做实验。而 $S$ 校有两年半的学制, 我有足 够的时间做研究与学习。”

\section{第三阶段：决定阶段}

当工科学生拿到不同大学的录取通知书时, 他们就进入了最终的决定阶段。 他们会主动地重新审视自己择校的整个过程, 使用成本收益分析的方法衡量在母 国学习、去他国学习、来中国学习的利弊得失。来自孟加拉国的 Ben 说:

“因为我们国家科研不是很好, 我一开始就确定要出国留学。我申请了中国、 土耳其、俄罗斯的高校, $S$ 校最先给我提供奖学金, 之后土耳其的学校也给我提 供了奖学金。土耳其的高校也很好，和我母国的文化传统、饮食习惯都很相似， 但我希望体验一种不同的文化环境; 中国和孟加拉国的合作也越来越多, 我想学 好汉语可能未来会有更多的发展机会。所以最终我选择了 $S$ 校。” 
Porter 也提到: “最终决定来华留学我是下定了很大决心的, 我放弃了在巴 基斯坦的研究生学位, 放弃了在巴基斯坦的教职, 我相信来华留学可以让我学到 更多的工科知识与能力、可以让我未来做一个更好的老师。”

从上面的例子可以看出, 国际学生对于是否来华学习会就政治、文化、经济 方面因素进行充分考量。他们可能会有其他的选择, 比如在母国学习、去西方国 家学习、或者继续工作。他们在最终决定之前, 会衡量每一个决定的可能后果, 如果他们认为来华学习能够获得更大的收益, 他们会选择来华留学。其整体决策 过程如图 1 所示。

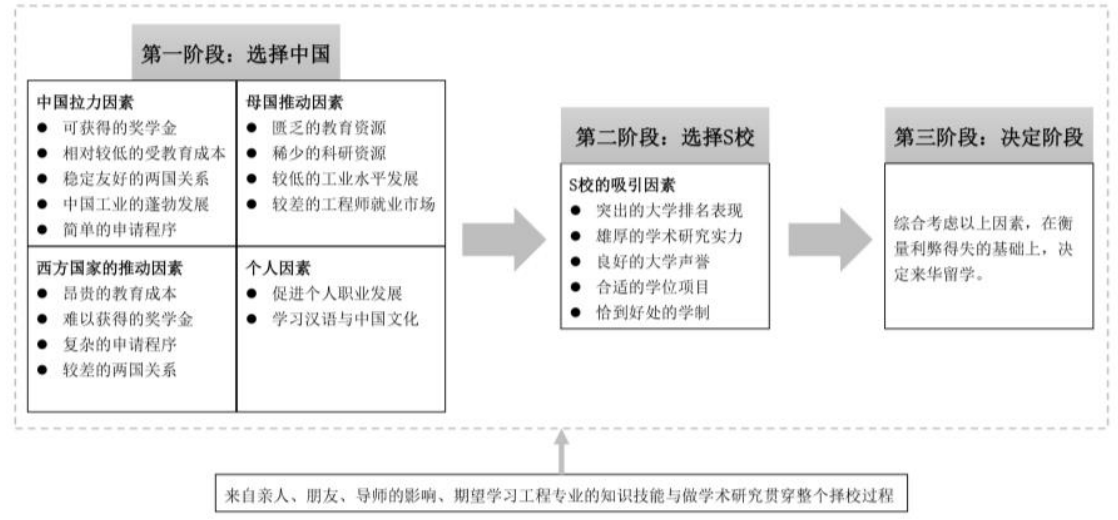

图 1. 工科学生来华留学择校过程

\section{讨论与建议}

由以上研究发现可以看出: 在影响来华留学的因素上, 工科学生认为可获得奖学 金、较低的受教育成本等经济因素, $\mathrm{S}$ 校全球大学排名表现突出、学术研究实力 雄厚等教育因素, 学习专业知识与技能、促进个人职业发展等个人因素是他们来 华留学的重要因素。本研究的发现与 Mazzarol 与 Soutar（2002）、Chen（2007） 等研究相一致: 即较为落后国家学生前往较为发达的国家留学, 更看重东道国的 教育质量; 此外, 本研究还发现来自法国和美国留学生在择校时几乎没有提及奖 学金等经济因素, 更多地强调希望体验一种新的生活方式、学习汉语和中国文化。 这可能从侧面说明来自发达国家和发展中国家的学生留学动机可能不同, 前者注 重个人兴趣, 后者注重经济因素（Ma, 2017）。在来华留学的择校过程上, 工科 学生的择校过程较为复杂, 每个阶段都受到了各种因素盘根错节的影响。与 Chen（2007）、Ma（2017）的研究发现一致, 留学生的择校过程是非线性的, 决定是否留学与留学哪里的问题 (Mazzarol \& Soutar, 2002) 交织在本研究的第一 阶段中。学生甚至可能跳过择校的第一阶段, 直接选择国外某一高校。然而, 本 研究未明显发现 Ma（2017）增添的笁选城市这一步骤, 可能因为工科研究生更 重视高校的综合实力与排名表现、合适的学位项目, 城市影响相对较小。此外, 作为小规模质性研究, 样本数量并未涉及其他城市, 这也可能导致研究发现具有 一定局限性。

总之, 本研究整体上表明中国工程教育实力日渐雄厚, 未来可能会吸引更多 的工科学生来华留学。然而, 在访谈中也发现了一些问题。例如, 针对留学生的 
英文授课课程较少, 英文授课质量不佳; 工科留学生获取留学信息的渠道单一, 多为朋友推荐等。综合以上分析讨论, 本研究提出以下建议:

\section{增加英文授课课程, 提高教师队伍专业水平}

中国工程院校在全球大学排名上的卓越表现和 2016 年加入华盛顿协议, 是 吸引工科学生来华留学的重要引擎。但突出的排名表现、雄厚的科研实力并不等 同于卓越的教学质量。很多工科学生提到他们缺乏编程等英文授课的专业课程, 教师难以清楚地使用英语讲解复杂的数学与工程知识。因此, 在英文课程的设置 方面, 中国可以借鉴日本 “超级全球化大学计划”（吴娴, 2017），选择国内试 点高校, 通过政策支持增加高校英文授课的课程数量、加强教师培训、增强其英 语授课能力等途径提升教学质量, 改善工科留学生的就读体验。

\section{加大海外招生宣传力度，优化招生手段}

大部分受访者提到他们来华留学的消息源是朋友、同事，一些受访者还表示 母国学生对中国院校概况几乎一无所知, 亦有研究发现留学生在获取择校信息的 时候会遇到很多困难（孙慧莉 \& 李忠明, 2017）。为了扩大中国工程院校的国际 知名度和影响力, 一方面应加大海外招生宣传力度，促使政府、民间、高校通力 合作，通过政府宣传、民间交流、师生交换、国际教育展、孔子学院等多种方式 向世界展示中国蓬勃发展的工程教育; 另一方面针对不同国家的招生宣传应该因 地制宜, 借鉴生源国招收外国学生的方法, 使招生理念与方法更能为他国学生所 接受，减少因招生宣传的理念和方法而产生的隔阂。

\section{发挥奖学金杜杆作用, 吸引高层次工科留学生}

已有研究表明中国来华留学生教育存在生源质量不理想的问题（陈强＆文 雯, 2018; 程伟华, 张海滨, \& 董维春, 2019）。数据显示：2016 年赴美留学的工科 学生接受研究生及以上学历教育的比例高达 $50 \%$ 以上, 而来中国的留学生接受研 究生教育的比例仅占比 20\%左右（Pew Research, 2017; 教育部国际合作与交流司, 2017）。结合可获得奖学金是工科学生来华留学的重要动机这一研究发现, 本研 究建议完善政府奖学金、学校奖学金、企业奖学金的资助制度, 使奖学金向高层 次人才倾斜的同时提升奖励额度, 更好地发挥奖学金的杜杆调节作用, 以吸引各 国优秀的工程人才来华留学, 优化工科来华留学生的生源结构。

\section{参考文献}

Altbach, P. G. (1998). Comparative higher education: Knowledge, the university, and development. Hong Kong, China: Comparative Education Research Centre, University of Hong Kong.

Chao, C., Hegarty, N., Angelidis, J., \& Lu, V. F. (2017). Chinese students' motivations for studying in the United States. Journal of International Students, 7(2), 257-269.

Chen, L. H. (2007). Choosing Canadian graduate schools from afar: East Asian students' perspectives. Higher Education, 54(5), 759-780. 
Chen, T. M., \& Barnett, G. A. (2000). Research on international student flows from a macro perspective: A network analysis of 1985, 1989 and 1995. Higher education, 39(4), 435-453.

De Wit, H., \& Altbach, P. G. (2018). Dramatic instability in international higher education. $\quad$ Retrieved October $10, \quad 2019$ from http://www.insidehighered.com/blogs/world-view/dramatic-instabilityinternational-higher-education

Ding, X. (2016). Exploring the experiences of international students in China. Journal of Studies in International Education, 20(4), 319-338.

Institute of International Education. (2015-2018). Global mobility trends. Retrieved October 10, 2019 from https://www.iie.org/Research-and-Insights/ProjectAtlas/Explore-Data

Lee, E. S. (1966). A theory of migration. Demography, 3(1),47-57.

Lu, G., \& Tian, M. (2018). An analysis of factors influencing international students' choice of education in China. In F. Dervin, X. Du, \& A. Härkönen (Eds.), International Students in China: Education, Student Life and Intercultural Encounters (pp. 15-46). Cham, Switzerland: Palgrave Macmillan.

Ma, J. (2017). Why and how international students choose Mainland China as a higher education study abroad destination. Higher Education, 74(4), 563-579.

Mazzarol, T., \& Soutar, G. N. (2002). "Push-pull" factors influencing international student destination choice. International Journal of Educational Management, $16(2), 82-90$.

Nghia, T. L. H. (2019). Motivations for Studying Abroad and Immigration Intentions. Journal of International Students, 9(3), 758-776.

Organization for Economic Co-operation and Development. (2018). What is the profile of internationally mobile students? Retrieved from http://www.oecd.org/education/education-at-a-glance

Pew Research. (2017). New foreign student enrollment at U.S. colleges and universities doubled since Great Recession. Retrieved from http://www.pewresearch.org/facttank/2017/11/20/new-us-foreign-student-enrollment-doubled-since-greatrecession

Ruby, A., (2009). The uncertain future for international higher education in the AsiaPacific Region. Presented at the invitation forum on new approaches to cooperation with Asia and Australia. NAFSA, Association of International Educators Annual Conference. Los Angeles, California.

Strauss, A., \& Corbin, J. M. (1990). Basics of qualitative research: Grounded theory procedures and techniques. Thousand Oaks, CA: Sage Publications, Inc.

Wen, W., \& Hu, D. (2018). The emergence of a regional education hub: Rationales of international students' choice of China as the study destination. Journal of Studies in International Education, 23(3), 303-325.

陈强, \& 文雯. (2018). “一带一路”倡议下来华留学生教育:使命、挑战和对策. 高校 教育管理, 12(3), 28-33.

程伟华, 张海滨, \& 董维春. (2019). “双一流”建设背景下来华留学研究生教育质量 研究——基于学生发展理论.学位与研究生教育, 314(01), 68-75.

教育部国际合作与交流司. (2003-2018). 2002-2017来华留学生简明统计. 北京: 教 育部国际合作与交流司.

李立国, 陈岗, \& 赫文婧. (2015). 为什么留学中国? --以中国人民大学留学生调查 为例. 复旦教育论坛, 13 (1), 37-43. 
宋华盛, \&刘莉. (2014). 外国学生缘何来华留学--基于引力模型的实证研究. 高等 教育研究, 35(11), 31-38.

孙慧莉, \& 李忠明. (2017). 来华学历生择校影响因素及择校途径研究--以江苏省为 例. 高教探索, (8), 82-89.

吴娴. (2017). 日本高校全英语学位课程的研究及对中国的启示. 清华大学教育研究, 38(6), 81-88.

\section{作者简介（Author biography）}

张国洋，上海交通大学高等教育研究院硕士研究生。

GUOYANG ZHANG is a graduate student majoring in higher education at Shanghai Jiao Tong University, China. Her research interests include project-based learning, and international engineering students' learning experiences and learning outcomes.

朱佳斌 (通讯作者), 副研究员, 上海交通大学高等教育研究院教育教学与人才成 长中心主任助理、IEEE Transactions on Education 副主编。电子邮箱: jiabinzhu@sjtu.edu.cn

JIABIN ZHU (corresponding author) is Associate Professor and Assistant Director of the Center for Education and Human Development in the Graduate School of Education at Shanghai Jiao Tong University, China. She currently serves as an associate editor for IEEE Transaction on Education. Email: jiabinzhu@ sjtu.edu.cn 